\title{
Psicoprofilaxis Obstétrica: Actualización, definiciones y conceptos
}

\author{
Sabrina Morales Alvarado ${ }^{1,2, a, b}$, Alex Guibovich Mesinas ${ }^{3, c, d}$, Maribel Yábar Peña ${ }^{4, e}$
}

\section{RESUMEN}

La Psicoprofilaxis obstétrica (PPO), reconocida como una herramienta de prevención en la atención obstétrica, tiene un impacto positivo en la salud materna y perinatal. En un mundo gl obalizado, es necesario y conveniente uniformizar criterios, con la finalidad que el personal que integra el programa, pueda trabajar mejor, bajo los mismos conceptos, comprendiendo el mismo significado y objetivos de la PPO, para lograr un trabajo en equipo, con calidad y de esta manera, conseguir el máximo beneficio para las madres, bebés y su entorno.

La unificación de definiciones y conceptos, servirá para lograr una gestión ágil y armoniosa en los aspectos técnicos, administrativos y clínicos.

El presente artículo tiene como finalidad revisar y actualizar las definiciones y conceptos en PPO, para que sirva de instrumento de apoyo en la gestión y desarrollo de los programas de PPO tanto públicos como privados. (Horiz Med 2014; 14(4): 53-57)

Palabras clave: Psicoprofilaxis obstétrica, definiciones, conceptos. (Fuente: DeCS BIREME).

\section{Obstetric Psychoprophylaxis: Update, definitions and concepts}

\begin{abstract}
Obstetric Psychoprophylaxis (OPP), recognized as a prevention tool in obstetric care has a positive impact on maternal and perinatal health. In a globalized world, it is necessary and desirable to standardize criteria, in order that staff integrates the program can work better under the same concepts, understanding the very meaning and objectives of the OPP, for a team work with quality and there by achieve the maximum benefit for mothers, babies and their environment.

Unifying definitions and concepts, serve to achieve a smooth and harmonious management in the technical, administrative and clinical aspects.

This article aims to review and update the definitions and concepts in OPP, to serve as a support tool in the management and development of OPP programs both public and private. (Horiz Med 2014; 14(3): 53-57)
\end{abstract}

Key words: Obstetric psychoprophylaxis, definitions, concepts. (Source: MeSH NLM).

\footnotetext{
Consultora de proyectos de intervención en salud reproductiva. Perú

Instituto Dulce Espera, Lima-Perú

Hospital Nacional Arzobispo Loayza, Lima-Perú

Institut Santé de la Femme, Lima-Perú

Doctora en ciencias de la Salud

Presidenta de la Sociedad Peruana de Psicoprofilaxis Obstétrica

Médico Ginecólogo Obstetra

Profesor de FMH-Universidad Nacional Mayor de San Marcos

Licenciada en Obstetricia
} 


\section{INTRODUCCIÓN}

La Psicoprofilaxis Obstétrica (PPO) como herramienta fundamental en la atención integral, con certeza tiene un impacto relevante en la salud de las madres y bebés. Desde que el ilustre Profesor Doctor Roberto Caldeyro Barcia (Montevideo, 1921-1996), médico perinatólogo, impulsor y Director del Centro Latinoamericano de Perinatología (CLAP - OPS/OMS), Montevideo, Uruguay, demostró y difundió su valor, desde la década de los 70 , no hay dudas sobre los importantes beneficios maternos y perinatales que ofrece la PPO y que igualmente, beneficia al equipo de salud (1-7). En un mundo globalizado, es necesario y conveniente uniformizar criterios, con la finalidad que el personal que integra el programa, pueda trabajar mejor, bajo los mismos conceptos, comprendiendo el mismo significado y objetivos de la PPO, para lograr un trabajo en equipo, con calidad y de esta manera, conseguir el máximo beneficio para las madres, bebés y su entorno (8-12).

\section{Actualización: Nuevos contenidos}

Con el desarrollo de la PPO en los últimos años, se han incorporado nuevos conceptos que han permitido desarrollar una PPO con mayor criterio técnico y calidad, como en el caso de algunas situaciones clínicas, que requieren que el profesional a cargo, con su capacidad discrecional, ofrezca en cada sesión un contenido de acuerdo a las condiciones de cada usuaria, como sería el caso de una gestante con placenta previa, gestación múltiple, enfermedad hipertensiva, en edad extrema u otros), la cual se ha denominado PPO en situaciones especiales (13-20).

El concepto actual de la PPO implica que siempre será positivo que la gestante reciba la preparación aún al final de su gestación, ya que recibirá consejos y entrenamiento que le permitirán responder de mejor manera y colaborar en el momento de su parto, concepto que ha sido definido como PPO de emergencia.

La elevada cifra de gestación en adolescentes en Perú, $13.5 \%$, hace necesario que los establecimientos cuenten con capacidad resolutiva para brindar la PPO con adecuación cultural y enfoque integral a este grupo de usuarias. En junio de 2012, el Ministerio de Salud Ilevó a cabo una Jornada preventivo promocional "Salud Nueva Actitud en la Adolescencia", en la ciudad de Pucallpa, en la cual asistieron más de cinco mil personas y de las cuales alrededor de quinientas fueron gestantes adolescentes, todas recibieron información, consejería sobre su salud y una sesión demostrativa de PPO, con lo cual quedó demostrado lo importante que es desarrollar a partir de una labor permanente preventivo promocional, la necesidad de atender a este grupo de adolescentes, incluyendo la PPO, como parte de su atención integral (17-19).

La PPO al ser una preparación integral que contempla personalización en la atención de cada gestante, debe ser con criterio clínico, enfoque de riesgo, ética y humanización $(7,22-25)$.

Con esta revisión, se aportará una orientación básica, sencilla y ágil a los profesionales de la Obstetricia, quienes están a cargo de los programas de PPO, tanto público como privado, para que como instrumento de consulta rápida puedan desarrollar las sesiones con un orden lógico y de manera didáctica, siendo recomendable para la unificación de criterios en cada proceso de la preparación.

Con la unificación de definiciones y conceptos, se podrá lograr una gestión ágil, armoniosa y siguiendo procesos en orden lógico, en los aspectos técnicos, administrativos y clínicos, por lo que esta revisión y actualización servirá de instrumento de apoyo para el desarrollo de los programas de PPO tanto públicos como privados.

\section{CONCEPTOS Y DEFINICIONES $(1,4,5,7,8,12-18,25)$}

\section{CONCEPTOS GENERALES}

- $\quad$ Gestante.- Mujer en estado de gravidez, desde la fecundación hasta el parto.

- $\quad$ Factores de riesgo.- Característica o atributo cuya presencia se asocia con un aumento de probabilidades de padecer el daño, por ejemplo: la desnutrición en la gestante condiciona entre otras, niños de bajo peso al nacer, anemia materna; por lo tanto, la mal nutrición es un factor de riesgo que se asocia a patologías tanto materna como neonatales, resulta así ser un factor asociado que aumenta la probabilidad del daño.

- Equipo didáctico básico de Psicoprofilaxis Obstétrica (KIT-PPO).- Denominado también Kit de Psicoprofilaxis Obstétrica, es el conj unto de herramientas, piezas, objetos o productos que constituyen el material didáctico básico para facilitar la sesión de PPO. Está constituido por una colchoneta, dos almohadas y un cojín.

- Gimnasia gestacional (GG)).- Actividad física a través de ejercicios gestacionales(EG) y movimientos generales y/o localizados que realiza la gestante para lograr bienestar general, entre ellos: refuerzo de músculos, elasticidad de tejidos, flexibilidad de articulaciones, mejor oxigenación tanto pulmonar como celular, comodidad, entre otros. La gimnasia gestacional se debe realizar con prudencia y de acuerdo a las condiciones de la gestante.

- $\quad$ Movimiento (Mv).- Cambio de posición del cuerpo o alguna zona del cuerpo respecto de una postura anterior.

- Posición inicial (PI).- Manera de acomodarse 
físicamente o postura adoptada antes de iniciar un movimiento, ejercicio o técnica.

- Posiciones aconsejables (PAc).- Posturas recomendables para la madre por prevenir y reducir dificultades, incomodidades o perjuicios durante la evolución de su gestación.

- Posiciones antálgicas (PAn).- Posturas que reducen las molestias, incomodidad o dolor en el cuerpo 0 algunas de sus partes.

- Posiciones prohibidas (PPh).- Posturas no recomendables para la madre por sus probables riesgos o perjuicios.

- $\quad$ Sesión de PPO (S\#).- Unidad de medida de cada atención de un programa de PPO en la cual se desarrolla el contenido de la preparación integral a la gestante/ pareja gestante. Se denomina también: Consulta de PPO, dado que implica un diagnóstico obstétrico y un tratamiento de PPO individualizado de acuerdo a las condiciones exhaustivas de cada gestante.

\section{PSICOPROFILAXIS OBSTÉTRICA}

- $\quad$ Psicoprofilaxis obstétrica (PPO).- Es la preparación integral, teórica, física y psicoafectiva, que se brinda durante la gestación, parto y/ o postparto para alcanzar una jornada obstétrica en las mej ores condiciones saludables y positivas en la madre y su bebé(s), forjándoles una experiencia satisfactoria y feliz; también fortalece la participación y rol activo del padre. La PPO debe ejercerse con criterio clínico, enfoque de riesgo y de forma personalizada, humanística y ética. La PPO disminuye las complicaciones y brinda una mejor posibilidad de una rápida recuperación, contribuyendo a la reducción de la morbilidad y mortalidad maternal perinatal.

- $\quad$ PPO Prenatal (PPO-PN).- Es la preparación integral, teórica, física y psicoafectiva, que se brinda durante la gestación. El mejor momento para iniciarla es entre el quinto y sexto mes prenatal; Sin embargo, hay que tener presente que nunca es tarde para realizarla.

- $\quad$ PPO Intranatal (PPO-IN).- Esla preparación integral, teórica, física y psicoafectiva, que se brinda durante el trabaj o de parto. Por el período en que se realiza, priorizará la información técnicas de ayuda a la madre y persona que le brinde el acompañamiento, enfocado hacia una respuesta adecuada durante la labor. También es denominada PPO de emergencia.

- $\quad$ PPO Postnatal (PPO-PTN).- Es la preparación integral, teórica, física y psicoafectiva, que se brinda después del parto. Por lo general se da durante el período de puerperio mediato, pudiendo ampliarse hasta los primeros meses del período de lactancia exclusiva. Su finalidad es lograr la recuperación y reincorporación óptima de la madre promoviendo cuidados en su salud sexual y reproductiva.

- $\quad$ Psicoprofilaxis de emergencia (PPO-EM).- Es la preparación brindada a la gestante faltando pocos días para la fecha probable de parto, o la que se ofrece a la parturienta durante su laborde parto. Es importante la adaptación de un programa o sesión intensiva teniendo en cuenta las prioridades. Busca esencialmente la tranquilidad, colaboración y participación de la gestante/parturienta para un mejor control emocional. La principal herramienta de la psicoprofilaxis de emergencia proviene de la escuela soviética ya que busca el "automatismo de la respuesta condicionada" para facilitar una mejor actitud, rol activo y experiencia obstétrica en la madre.

- Psicoprofilaxis Obstétrica en situaciones especiales (PPO-SE).- Preparación prenatal integral que se da a las gestantes cuya gestación no reúne las condiciones normales y/o ideales, por lo que de acuerdo con cada situación especial (SE), requerirá una atención basada en las necesidades específicas de cada madre. Por su origen, la SE puede ser de tipo personal, médica o sociocultural.

- $\quad$ Preparación física (PF).- Entrenamiento que se brinda a la gestante de acuerdo con sus condiciones generales y obstétricas para que obtenga beneficios corporales y mentales. La prepara para lograr una gestación, parto y recuperación en mejores condiciones. Ésta puede ser a través de ejercicios gestacionales, técnicas de actividad respiratoria, técnicas de relajación neuromuscular, posiciones antál gicas y/ o masaj es localizados, entre otros. - $\quad$ Preparación psicoafectiva (P-PA).- Apoyo emocional que se brinda a la gestante/ parej a gestante, permitiéndole disfrutar la llegada del bebé sin temores o ideas negativas, logrando en ellos tranquilidad, seguridad y bienestar permanentes. Ayuda también en el proceso de desligar el fenómeno de temor - tensión - dolor. La preparación psicoafectiva circunscribe la Estimulación Prenatal.

- Preparación teórica (PT).- Información real y oportuna que se brinda a la gestante para que conozca y entienda aspectos relacionados con su jornada obstétrica, lo cual la ayuda a tener tranquilidad y seguridad, empoderándola respecto de los cuidados de salud y generando en ella una cultura de prevención.

\section{VÍNCULO AFECTIVO Y ESTIMULACION PRENATAL}

- Estimulación.- Es la transferencia de una acción que logra una respuesta a partir de los órganos de los sentidos.

- $\quad$ Estímulo.- Agente o causa que provoca una reacción o una respuesta en el organismo o en una parte de él.

- Estimulación intrauterina (EIU).-Es el uso de procesos, métodos, instrumentos y/ o técnicas diversas con la finalidad de lograr una respuesta real y objetiva en el embrión/feto. Clínicamente la EIU es empleada con fines diagnósticos. Ejemplo: estimulación vibro acústica para facilitar pruebas de bienestar fetal.

- $\quad$ Patrones de reactividad fetal.- Es la observación de la respuesta objetiva de las reacciones del feto intraútero mediante el uso de la tecnología como equipos ecográficos, para apreciar su respuesta frente a algún estímulo intrauterino.

- Estimulación Prenatal (EPN).- Acciones que 
promueven y motivan en la madre el vínculo afectivo durante la gestación, propiciando sentimientos de acogida, afecto y bienestar respecto de la llegada del hijo o hija. El vínculo afectivo prenatal comprende un contacto activo con su entorno inmediato, en particular con la pareja, hijos y personas más allegadas. La Estimulación Prenatal es parte de la preparación psico-afectiva de la Psicoprofilaxis Obstétrica. Se han descrito en algunas fuentes, ciertas técnicas de intervención a manera de "estímulo intrauterino" (visual, táctil, motora, auditiva), sin embargo, por carecer de suficiente sustento científico no se consideran ni se incluyen en el componente educativo ni en otra área de la preparación prenatal.

- Vínculo prenatal (VPN).- Lazo afectivo que se propicia en la gestante/pareja gestante respecto de la llegada de su hijo o hija, para que su llegada sea asumida de manera natural, positiva, con acogida y afecto.

- $\quad$ Apego.- Es la relación y vínculo afectivo estrecho que desarrolla el niño o niña con sus padres, lo cual influirá en su seguridad emocional, necesarios para un buen desarrollo de su personalidad.

\section{MÉTODOS Y TÉCNICAS}

- Técnicas de relajación (TRj).- Son las diferentes modalidades de lograr un estado de reposo tanto físico como mental en la madre, con la finalidad de hacerle ahorrar energía, lograr alivio de tensiones o molestias y mejorar su capacidad de concentración. Las técnicas de relajación pueden ser de manera profunda o progresiva (técnica de Jacobson y Shultz), utilizadas como técnicas básicas en sofrología, para un mejor autocontrol de las molestias.

- Técnicas de respiración (TRs).- Son las diferentes modalidades de efectuar la oxigenación pulmonar, la que a su vez mejora la oxigenación celular en la madre y el bebé, dependiendo del momento y la necesidad de la gestante o parturienta, con lo cual logrará también mayor comodidad, tranquilidad, facilitando la relajación y control de las molestias propias de cada fase de la gestación o trabajo de parto.

- $\quad$ Técnicas de vinculación prenatal (TVpn).- Acciones o dinámicas que buscan propiciar el lazo afectivo en la gestante/ pareja gestante respecto de la llegada de su hijo o hija, para éstasea asumida de manera natural, positiva, con acogida y afecto.

- Calistenia (Clt).- Movimientos suaves, rítmicos y progresivos que se utilizan en especial al inicio de la preparación física de la gestante con la finalidad que logre un estiramiento natural para una mayor comodidad, elasticidad y flexibilidad durante los movimientos del entrenamiento físico.

- Visualización.- Procedimiento mediante el cual se crea mentalmente imágenes construidas en base a la motivación y pensamientos especialmente durante la relajación, para lograr un estado emocional más agradable, placentero y reconfortante.

\section{MÉTODOS ALTERNATIVOS}

- $\quad$ Aromaterapia.- Es un método alternativo mediante el uso de fragancias a través de aceites esenciales aromáticos (aceites extraídos de hojas, flores, troncos y raíces), con la finalidad de promover la salud y el bienestar del cuerpo, la mente y las emociones. El uso de estas esencias mediante diversos sistemas de aplicación puede restablecer el equilibrio y la armonía en la gestante, estado que beneficia al bebé.

- Esferodinamia.- Es una modalidad de entrenamiento físico con el uso de una esfera o pelota de plástico inflada con aire, con la finalidad de facilitar algunos movimientos y posturas que faciliten la preparación física prenatal y postnatal en la madre.

- Cromoterapia.- Es el tratamiento de diferentes patologías utilizando como agente físico terapéutico la interacción de longitudes de onda en regiones seleccionadas del espectro electromagnético con los sistemas biológicos. Las regiones de interés son la ultravioleta cercana, visible e infrarrojo cercano, que constituyen las radiaciones electromagnéticas no ionizantes.

- Masoterapia.- Es un método alternativo mediante el uso de manipulaciones de los tejidos blandos del cuerpo ejercida especialmente con las manos, como modo más eficaz, cuya finalidad es lograr principalmente el alivio de molestias, relajación, mejorar la circulación y oxigenación, además de favorecer un descanso adecuado, el estado emocional y la disminución de la fatiga tanto física como emocional en la gestante o puérpera.

- Matronatación Prenatal.- Es el uso del agua como recurso para facilitar la preparación prenatal, en especial en el área física, y que por sus características y beneficios, como la disminución de la gravedad, facilita los movimientos, flexibilidad y reduce el esfuerzo físico, asimismo, por el efecto de hidromasaje homogéneo en todo el cuerpo de la gestante, reduce molestias y genera sensación de bienestar integral, entre muchos otros beneficios. La matronatación, hay que indicarla con criterio clínico, considerando sus contraindicaciones relativas y absolutas.

- Musicoterapia.-Es un método alternativo terapéutico mediante el uso de estructuras musicales, tonos, sonidos, melodías y otros, con la finalidad de lograr motivación, relajación, meditación y estimulación durante la sesión de psicoprofilaxis obstétrica, logrando así un mej or estado psico-físico, optimizando el estado emocional, cognitivo y espiritual en la gestante o puérpera y su pareja durante su preparación.

- $\quad$ Sofrología. - Es una escuela científica que estudia la conciencia, sus modificaciones y los medios físicos, químicos o psicológicos susceptibles de modificarla, con una finalidad terapéutica, profiláctica o pedagógica en medicina.

\section{Agradecimientos}

Nuestro agradecimiento al equipo profesional, por su tiempo en la contribución de este trabajo de revisión. 


\section{REFERENCIAS BIBLIOGRÁFICAS}

1. ASPPO (Soc. Peruana de Psicoprofilaxis Obstétrica). Manual de Psicoprofilaxis Obstétrica, 3ra Ed. Lima: ASPPO. 2010.

2. MINSA (Ministerio de Salud - Perú). Normas técnico administrativas para la atención integral de la salud materno perinatal. Lima: MINSA. 1995.

3. MINSA (Ministerio de Salud - Perú).Psicoprofilaxis ObstétricaMódulos de capacitación, Proyecto 2000, OPS/OMS. Lima: MINSA. 1997.

4. Equipo Técnico ASPPO. Preguntas y respuestas sobre la estimulación prenatal. Boletín Científico ASPPO 2006; 8(23): 8

5. MINSA. Guía técnica para la Psicoprofilaxis Obstétrica y Estimulación prenatal. Lima. 2011.

6. MINSA. Norma técnica para la atención del parto vertical con adecuación intercultural. Lima: MINSA. 2005.

7. Morales S. La Psicoprofilaxis Obstétrica como eje de la prevención prenatal, $1^{\mathrm{a}}$. ed. Lima: Cimagraf. 2004.

8. Gavensky R. Parto sin temor y parto sin dolor, 17ma. Ed. Buenos Aires: El Ateneo. 1987.

9. EsSalud. Guía de Estimulación prenatal. 1ra. Ed. Lima, Perú. 2006.

10. Ministerio de Salud y Ambiente de la Nación. Preparación integral para la maternidad. Argentina. 2005.

11. Videla M, Grieco A. Parir y Nacer en el Hospital. 1ra Ed. Buenos Aires: Nueva Visión; 1993.

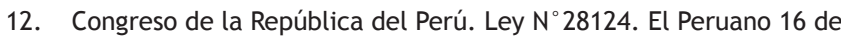
Diciembre 2003.

13. Pecho S., Reflexiones acerca de la Estimulación Prenatal (EPN) y el consentimiento informado como indicadores de la calidad de atención en salud. Boletín Científico ASPPO 2014; 16(47): 8-9.

14. Morales S. La Psicoprofilaxis Obstétrica como eje de la prevención prenatal, $1^{\text {a }}$. ed. Lima: Cimagraf. 2004. 176.

15. Morales S. Psicoprofilaxis Obstétrica - Guía práctica, 1ra. Ed. Lima: ASPPO. 2012.

16. Morales S. Psicoprofilaxis obstétrica con ciencia y calidad. Boletín Científico ASPPO 2012; 13(39): 4-5.

17. Morales S. Impacto de la Psicoprofilaxis Obstétrica en la reducción de la morbilidad y mortalidad materna y perinatal. Horiz Med 2012; 12(2): 47-50.

18. Morales S., García Y., Agurto M., Benavente A., La Psicoprofilaxis Obstétrica y la Estimulación Prenatal en diversas Regiones del Perú. Horiz Med 2014; 14(1): 42-48.

19. Ministerio de Salud. Salud Nueva Actitud, Memoria de gestión 2011- 2012. Lima 2012.

20. CLAP - OPS/OMS. Guías para el continuo de atención de la mujer y el recién nacido. Publicación científica $\mathrm{N}^{\circ}$ 1577. Cap. 3, 2011. 298.CLAP - OPS/OMS. Boletín de Salud Perinatal 2000; 18: 1-44.
21. March of Dimes Birth Defects Foundation: Toward Improving the Outcome of Pregnancy - The 90s and Beyond. White Plains, NY: The March of Dimes Birth defects Foundation, 1993.

22. OPS/OMS. Salud para todos en el año 2000. 1980.

23. American College of Obstetricians and Gynecologistis. Exercise during pregnancy and the postpartum period. ACOG Technical Bulletin $\mathrm{N}^{\circ}$ 189. Washington DC, ACOG Press, 1994.

24. OPS/OMS. Acciones de Salud Materno Infantil a Nivel Local: según las Metas de la Cumbre Mundial a favor de la Infancia. 1996.

25. Verny T. El vínculo afectivo con el niño que va a nacer. España: Urano; 2011.

\section{Fuentes de financiamiento}

Este estudio ha sido parcialmente financiado por la Sociedad Peruana de Psicoprofilaxis Obstétrica y parcialmente autofinanciado por la autora principal.

\section{Contribuciones de autoría}

Este fue un estudio donde la autora participó en la recolección y análisis de datos y el proceso de desarrollo del manuscrito.

\section{Conflictos de interés}

Los autores declaran no tener ningún conflicto de interés.

\section{Correspondencia:}

Sabrina Morales Alvarado

Dirección: Av. Javier Prado Oeste 555 Dpto. 1501 Lima 27, Perú.

Teléfono: +(51 1) 421-0979 / 999-096282

Correo electrónico: sabrynes@gmail.com /

sabrinamorales@asppo.org
Recibido: 20 de Junio de 2014 Aprobado: 01 de Agosto de 2014 


\section{REVISTA HORIZONTE MÉDICO \\ NORMAS PARA PUBLICACIÓN}

Horizonte Médico es editada por la Facultad de Medicina Humana de la Universidad de San Martín de Porres (USMP). Tiene por finalidad difundir el conocimiento científico principalmente médico, entre los profesores y alumnos de la universidad y de otras universidades, profesionales de disciplinas afines a la Medicina y personas interesadas.

Para la presentación de trabajos, no es necesario ser profesor o alumno de la USMP, sino un profesional o alumno de las ciencias bio-psico-médico-sociales de cualquier universidad, que desarrolle artículos originales e inéditos.

\section{Tipos de trabajos:}

Los trabaj os que se presenten a la revista pueden ser de los siguientes tipos:

$\begin{array}{ll}\text { - } & \text { Artículo original } \\ \text { - } & \text { Original Breve } \\ \text { - } & \text { Tema de revisión } \\ \text { - } & \text { Caso clínico } \\ \text { - } & \text { Comunicación corta } \\ \text { - } & \text { Historículo de opinión } \\ \text { - } & \text { Semblanza }\end{array}$

\section{NORMAS PARA LA PRESENTACIÓN DE TRABAJOS}

La revista Horizonte Médico se edita de acuerdo a los "Requerimientos uniformes para los manuscritos remitidos a las Revistas Médicas" y las Normas de Vancouver.

Normas Generales:

Los trabaj os enviados para su publicación deben cumplir las siguientes normas de presentación:

- Tratar temas relacionados al área bio-psicomédico-social de la Salud.

- Ser originales e inéditos.

- $\quad$ Pertenecer a una de las siguientes categorías:

Artículo original: Trabajo de investigación inédito sobre una materia relacionada con el campo científico, técnico, humanístico o ético deontológico de la Medicina.

Original Breve: Son productos preliminares de investigaciones en curso o informes de brotes que por su importancia merecen ser difundidas. Tiene la siguiente estructura: resumen no estructurado, palabras clave, introducción, el estudio (que incluye "hallazgos" para mostrar los resultados), discusión y referencias bibliográficas (límite: 150 palabras resumen, 2000 palabras de contenido, cuatro figuras o tablas y veinte referencias).

Tema de revisión: Sintetiza, analiza y actualiza un tema del campo de la medicina, incluyendo las evidencias científicas; por ejemplo: la acción de la prostaglandina E2 en la maduración del cuello uterino.

Caso Clínico: Discusión de un caso de la clínica médica o quirúrgica de interés diagnóstico, de observación rara o de evidente interés que amerite su publicación, como sería la discusión del primer caso de Dengue en Lima.

Comunicación corta: Es un texto breve sobre un tema determinado, que el autor presenta para conocimiento y discusión del lector. Puede ser el relato breve preliminar de una investigación original en curso o ya terminada y en proceso de redacción del artículo final, o que no tiene la trascendencia de un trabajo original.

Artículo de opinión: Es un escrito que se caracteriza por la exposición y argumentación del pensamiento de una persona reconocida acerca de un tema relacionado con la medicina, como: Atención primaria de salud, enviada por el Representante de la OPS en el Perú.

Historia: Es la narración y exposición de acontecimientos pasados trascendentes en el campo de la medicina, como la historia de la primera cesárea segmentaria practicada en el Perú.

Semblanza: Bosquejo biográfico de un médico o personaje contemporáneo, cuya labor haya tenido particular influencia en el campo de la medicina o en el desarrollo de la enseñanza de esta disciplina en la USMP, en el Perú o en el mundo.

Carta al editor: Comunicación escrita corta dirigida al Editor de la Revista.

- Los trabajos serán redactados en español, impresos en papel bond blanco de medida estándar A4, en una sola cara, a doble espacio, con márgenes de por lo menos $25 \mathrm{~mm}$. Cada componente del manuscrito debe empezar en página aparte. Las páginas deben numerarse en forma consecutiva. Se debe entregar el original y una copia y enviar el trabajo debidamente grabado en medio magnético, en formato MS Word y/o enviar por correo electrónico.

- Se debe adjuntar una declaración jurada firmada por todos los autores, reconociendo que el artículo presentado es propiedad intelectual de los autores y que no ha sido publicado, ni presentado para evaluación en otra revista, cediendo los derechos de autor a Horizonte Médico una vez que el artículo sea aceptado para su publicación.

- Los trabajos serán evaluados por el Comité Editorial y sometidos a un arbitraje por pares, profesionales especializados que califican los trabajos a solicitud del Comité, mediante un informe acerca del mismo.

- La Revista Horizonte Médico se reserva el derecho de aceptar los trabajos presentados y de solicitar las modificaciones que considere necesarias para cumplir con las exigencias de la publicación. También, se reserva el derecho de uniformar el manuscrito de acuerdo al formato de la revista. 
Referencias bibliográficas

Las referencias bibliográficas corresponderán exclusivamente al texto del trabajo, ordenados correlativamente según su aparición, y se redactarán siguiendo las Normas de Vancouver. Deberán redactarse de la siguiente manera:

\section{- Artículos de revistas:}

Apellidos del autor y coautores, seguidos de la inicial del nombre. Si el número de autores es más de seis (6), se pone sólo el nombre del autor seguido por la palabra "et al" en latín o "y col" en español. Luego de los autores, se coloca un punto seguido y a continuación se cita el título del artículo en el idioma de origen. Termina en punto seguido.

A continuación el nombre de la Revista (en abreviatura reconocida internacionalmente) y el año de publicación, un punto y coma; el número de volumen, seguido de dos puntos, las páginas entre las que aparece el artículo y un punto final. Ej.: Sáenz C, Santana S. Cesárea electiva y parto vaginal en cesareadas previas: Comparación de complicaciones maternas neonatales. Rev Per Ginecol Obstet 2010;56:232237.

\section{- Libros, folletos o similares:}

Autor y coautores en igual forma que para los artículos. Título del libro, punto seguido y luego la preposición "En" (para capítulos) seguida de dos puntos, apellidos e iniciales de los nombres de los editores del libro seguida de la palabra "editor(es)", punto y el título del libro, en el idioma de origen; punto seguido y el número de edición, punto; lugar de la edición y dos puntos, nombre de la editorial, punto; año de la edición, dos puntos y (sin separación) páginas entre las que aparece el trabajo.

Ej.: Acha P, Szyfres B. Zoonosis y enfermedades transmisibles comunes al hombre y los animales. 3a ed. Washington DC. Organización Panamericana de la Sa lud; 2003.

\section{- Tesis:}

Autor: En igual forma que para los artículos. Título del trabajo. Entre paréntesis, especificar el grado optado, punto seguido. Ciudad y país donde se sustentó, separado por una coma, dos puntos y la Universidad de procedencia, una coma, el año y punto seguido. El número de páginas, seguido de la abreviatura pp.

Ej: Pino-Ruiz LK. Asociación del Helicobacter pylori con las enfermedades esófago gastroduodenales en la Clínica Good Hope. Miraflores Período del 2009 a junio de 2010 (Médico Cirujano). Lima, Perú. USMP (Facultad de Medicina), 2011. $67 \mathrm{pp}$.

De los temas de revisión:

- Deben redactarse según el siguiente esquema:

- Resumen en un máximo de 250 palabras, además de las palabras clave en español.

- Abstract en un máximo de 250 palabras, además de las palabras clave en inglés (key words).

Desarrollo del tema.

- Referencias bibliográficas.
- En lo demás, se siguen las indicaciones para los artículos originales.

\section{De las comunicaciones cortas/caso clínico:}

- Deben redactarse según el siguiente esquema:

- Resumen con una extensión máxima de 125 palabras. Breve introducción, comunicación o caso(s) clínico(s), discusión y referencias bibliográficas.

- La extensión total del trabajo, incluyendo referencias bibliográficas, no debe ser mayor de seis (6) páginas escritas en una sola cara. Se aceptarán como máximo cuatro figuras y/ o tablas.

\section{De las cartas al Editor:}

- Deben redactarse según el siguiente esquema:

- Carta, referencias bibliográficas. Deben tener una extensión máxima de dos páginas. Se aceptarán como máximo dos figuras y/o tablas. El número máximo de autores será cinco y de referencias bibliográficas no más de 10.

\begin{tabular}{|c|c|c|c|}
\hline № & Consideraciones & $\begin{array}{c}\text { Imagen } \\
\text { digitalizada por } \\
\text { cámara } \\
\text { fotográfica }\end{array}$ & $\begin{array}{c}\text { Imagen } \\
\text { digitalizada } \\
\text { por escáner }\end{array}$ \\
\hline $1 \%$ & $\begin{array}{c}\text { Resolución } \\
\text { (igual o mayor) }\end{array}$ & 3 megapixeles & 600 dpi o ppp \\
2 일 & $\begin{array}{c}\text { Formato o } \\
\text { extensión }\end{array}$ & JPG, BMP o TIF & JPG, BMP o TIF \\
\hline
\end{tabular}

\section{Consideraciones generales:}

- $\quad$ Figura: Son los dibuj os, mapas, fotografías, gráficos estadísticos o cualquier ilustración que no sea Tabla.

- Tabla: Deben tener sólo líneas horizontales para separar el encabezado del cuerpo de la tabla, en ningún caso deben incluir líneas verticales.

\section{Descargo de responsabilidad:}

La publicación de trabajos en Horizonte Médico, en sus diferentes secciones; no significa que, necesariamente, el Comité Editor se solidarice con las opiniones vertidas por él o los autores.

\section{Síguenos en:}

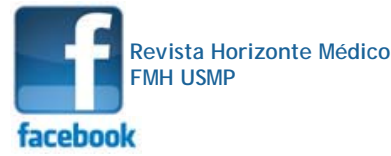

@HorizMed

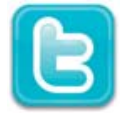

twittery 


\section{CALENDARIO DE EVENTOS}
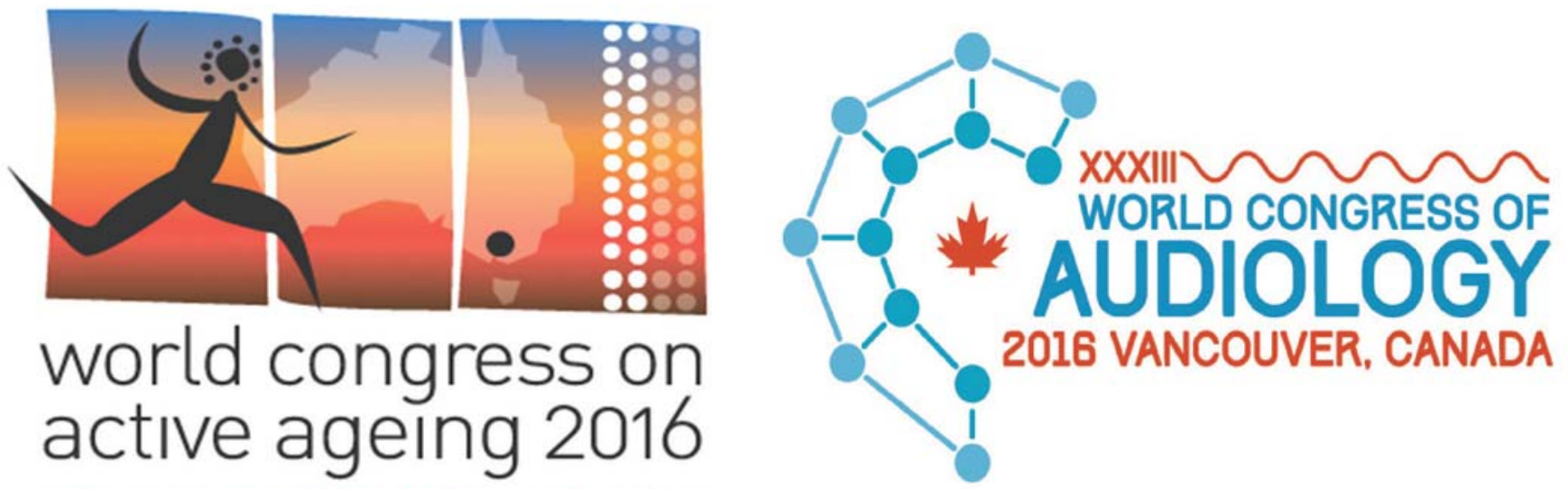

28 June - 1 July 2016 Melbourne Australia
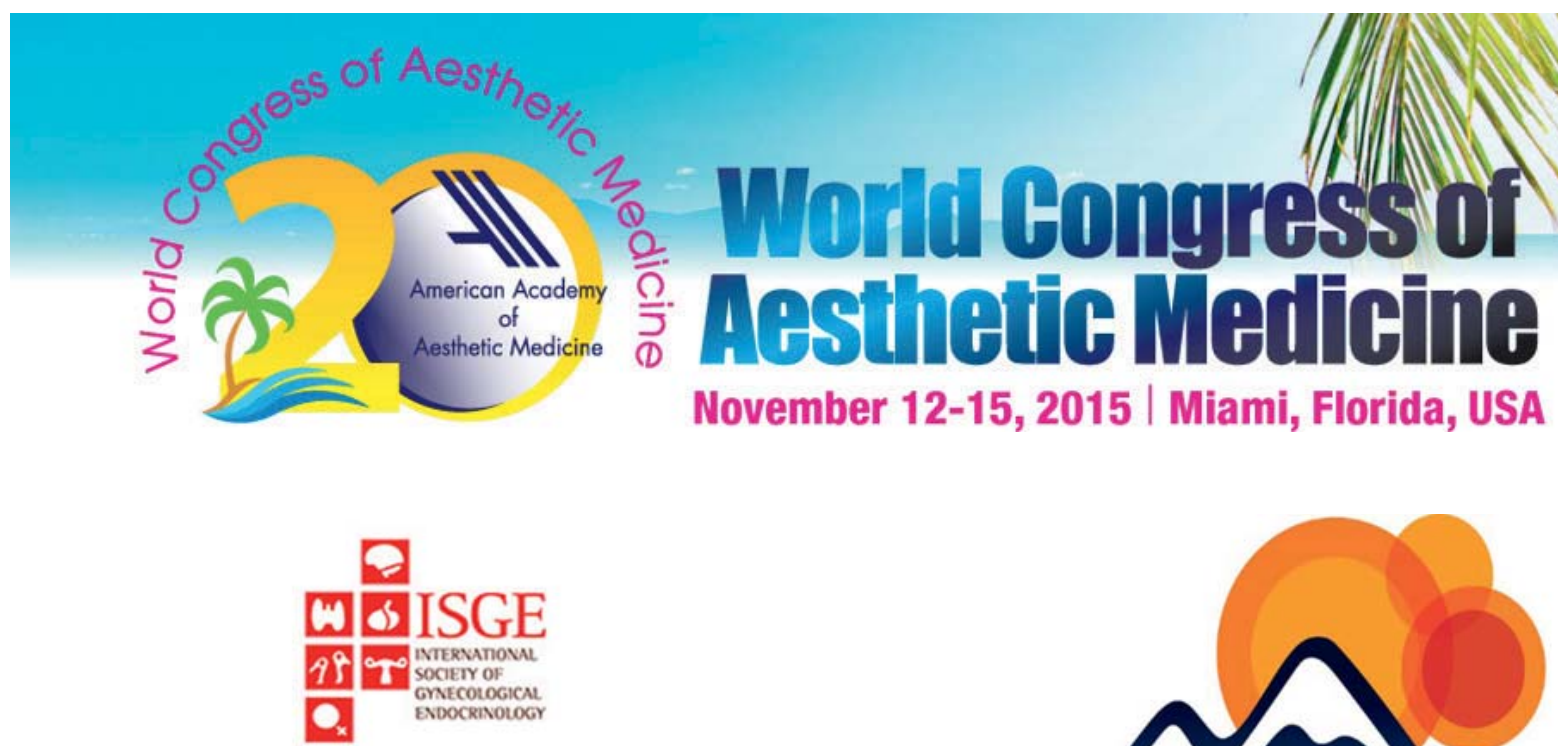

GYNeCOLOGICAL ENDOCRINOLOGY

THe 17 "' WORLD CONGRESS

MARCH 2-5 2016

FIRENZE, ITALY
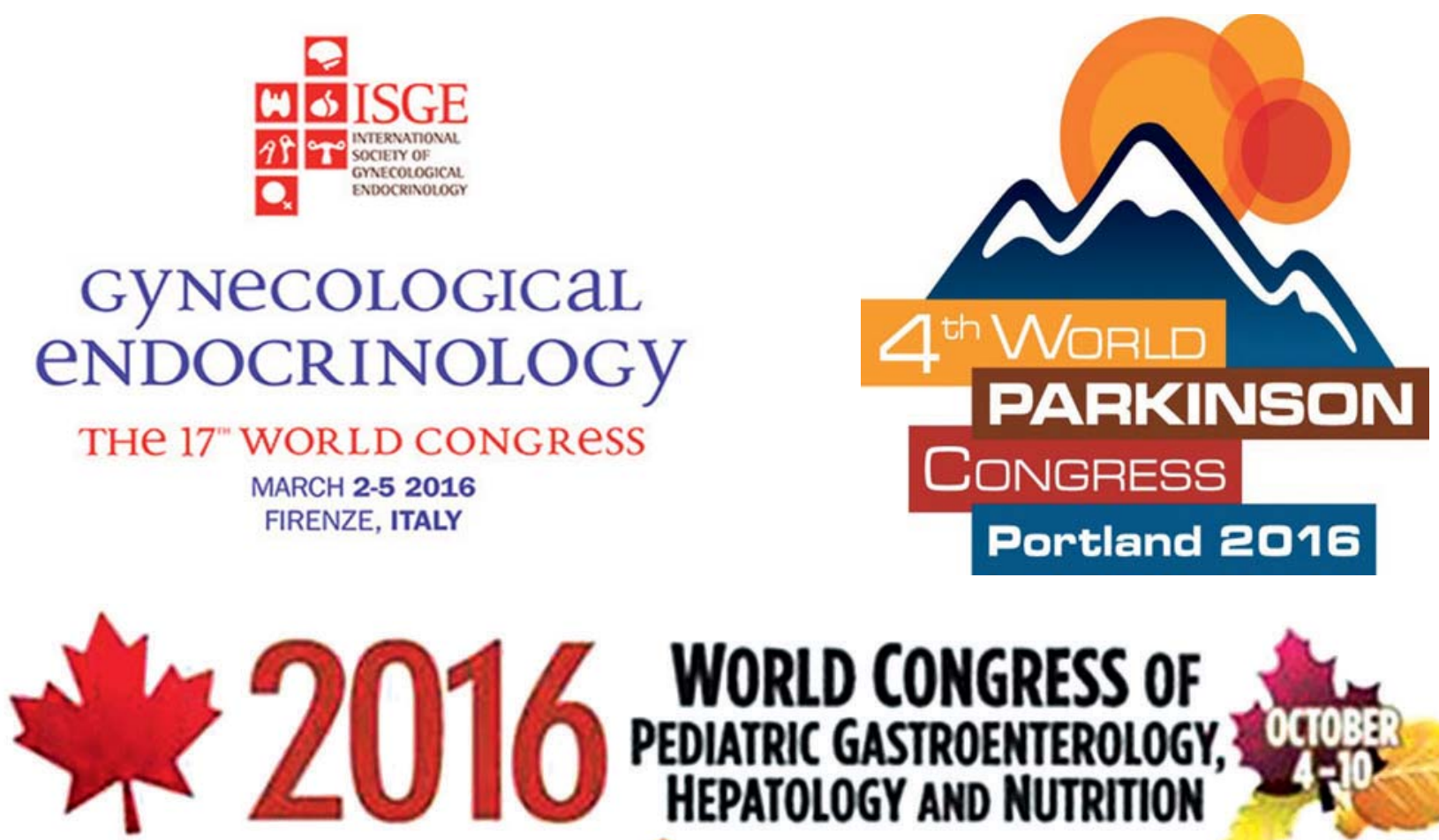

WORLD CONGRESS OF PEDIATRIC GASTROENTEROLOGY, OGOS:S HEPATOLOGY AND NUTRITION MONTRÉAL * CANADA 


\section{HORIZONTE MÉDICO}

Difundiendo la Investigación, más allá; de nuestras fronteras

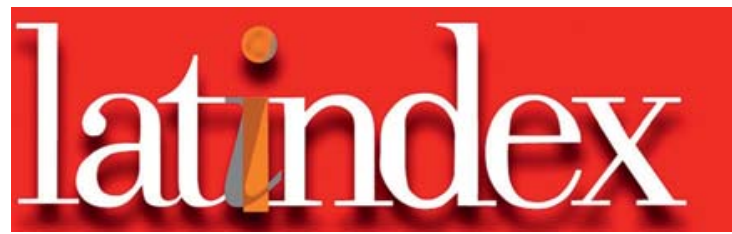

LILACS
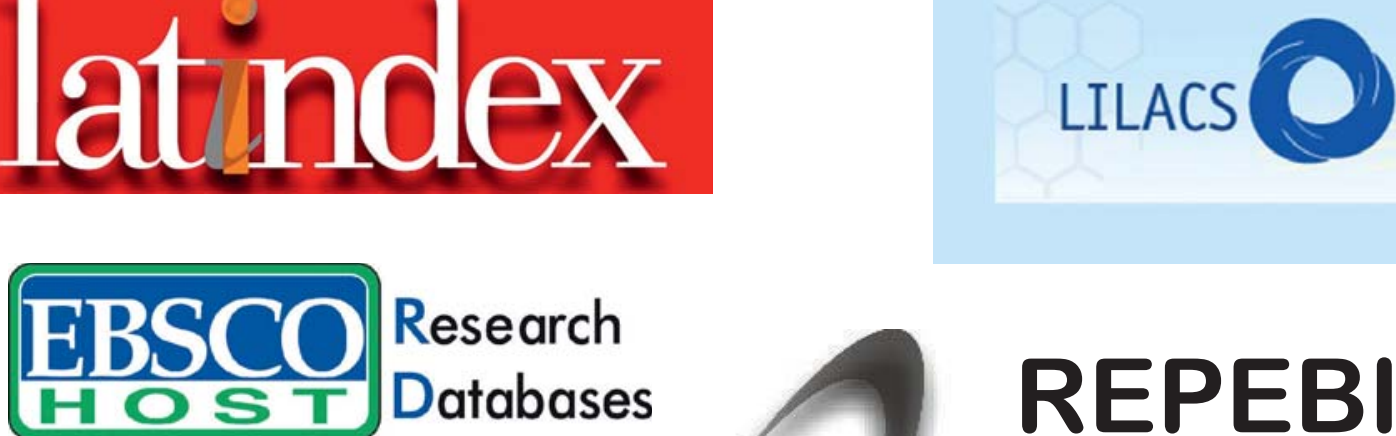

Research

Databases

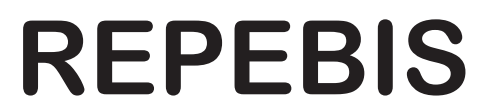

Red Peruana de Biblioteca en Salud

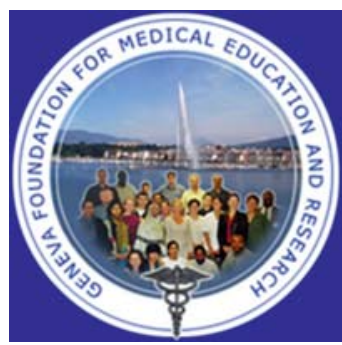

Scientific Electronic Library Online
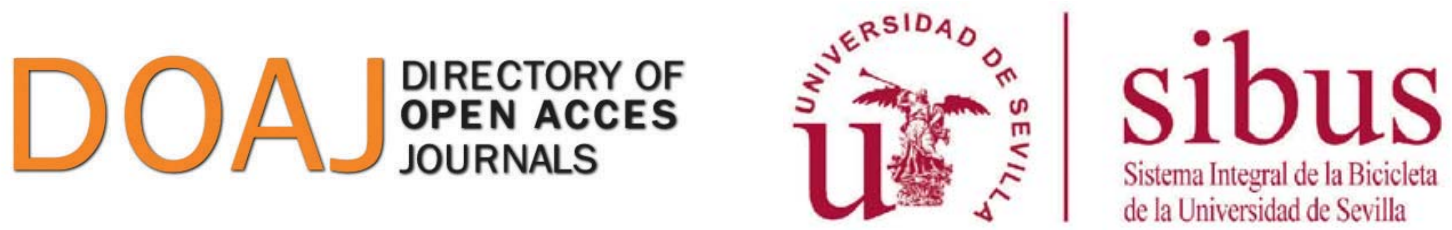

Dirección: Av. El Corregidor 1531, La Molina, Lima, Perú

Teléfonos: 365-2300, 365-2574, 365-3640 - Fax: 365-0487

horizonte_medico@usmp.pe

www. medicina. usmp. edu. pe/horizonte/principal, php 


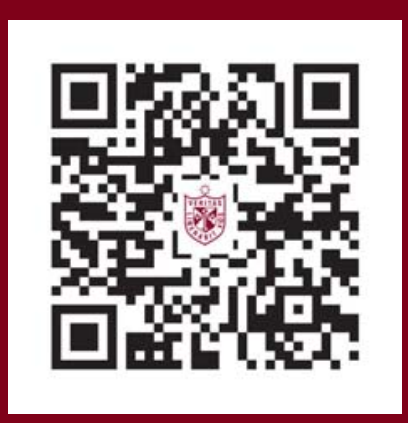

